\title{
Horticultural Factors Affecting Antioxidant Capacity of Blueberries and other Small Fruit
}

\author{
W. Kalt, ${ }^{1}$ A. Howell, ${ }^{2}$ J.C. Duy, ${ }^{1}$ C.F. Forney, ${ }^{1}$ and J.E. McDonald ${ }^{1}$
}

\begin{abstract}
Additional IndeX wORds. Vaccinium, phenolic, anthocyanin, flavonoids
Summary. It is now widely held that the antioxidants contained in fruit and vegetables can provide protection against certain human degenerative conditions that are associated with oxygen free radical damage. This view is supported by epidemiological, in vitro, and more recently, in vivo evidence. Phenolics (polyphenolics) contribute substantially to the antioxidant complement of many small fruit species whose ripe fruit are red, purple or blue in color. Fruit containing high levels of phenolic antioxidants would be attractive to health conscious consumers, therefore optimization of production and processing factors affecting small fruit antioxidant capacity is desirable. In many small fruit crops, antioxidant activity [measured as oxygen radical absorbing capacity (ORAC)] is positively correlated with their content of anthocyanins and total phenolics. Genera, species, and genotypes vary with respect to phenolic content. Both annual and geographical factors appear to influence ORAC, although many years of study are needed to distinguish these effects from other biotic and abiotic factors that influence fruit phenolic content. Antioxidant capacity due to phenolics is decreased by food processing practices, such as heat or aeration.
\end{abstract}

preponderance of epidemiological studies have indicated a negative corre-
lation between fruit and vegetable consumption and the risk of age-related
conditions including certain cancers, cardio- and cerebrovascular disease, and possibly neurodegenerative disease (see references in Ames et al., 1993). With respect to cancer, the review by Block et al. (1992) concluded a negative correlation between fruit and vegetable consumption and the incidence of various cancers, in 129 of 172 epidemiological studies. Steinmetz and Potter (1996) reported a similar trend in their review of 206 studies. A strong protective effect of fruit and vegetable consumption against stroke, and a weaker protective effect against coronary heart disease was reported by Ness and Powles (1997) in their retrospective review of several types of studies. Epidemiological evidence supported a protective role for fruit and vegetable consumption against ischemic stroke risk (Joshipura et al., 1999). Reduced mortality from ischemic heart disease and stroke was reported in a study of 11,000 health conscious individuals who consumed fruit daily (Key et al., 1996). Hertog et al. (1993) associated a decreased risk of coronary heart disease with the consumption of specific fruit and vegetable components, namely flavonoids. 
The basis for the protective effect of fruit and vegetables against human degenerative diseases is described in the widely cited paper by Ames et al. (1993). These authors discuss the ubiquitous and destructive nature of oxygen radicals and describe how oxygen radicals can cause damage to biomolecules like DNA, lipids, and proteins. They estimate that the DNA in each human cell sustains 10,000 oxidative hits per day. In spite of DNA repair mechanisms, oxidative lesions accumulate with age, and specific mutations give rise to cancer (Ames et al., 1993). Similarly, oxidation of low density lipoprotein is a significant factor leading to the development of vascular plaque in atherosclerosis (Fuller and Jialal, 1997). In neurobiology, oxidative stress and damage are under investigation with respect to normal brain aging as well as neurodegenerative disease. Several lines of evidence suggest an association between oxidative damage in the brain, and neurological decrements (Cantuti-Castelvetri et al., 2000).

How do fruit and vegetable components provide protection against the deleterious effects of oxidative stress? Phytochemicals found in fruit and vegetables have structural features and redox capacities that make them effective in combating oxygen free radicals via various mechanisms. Ames et al. (1993) present several arguments to support a protective role for fruit and vegetable antioxidants $(\mathrm{AO})$ in human health. Currently there is a relative abundance of epidemiological and in vitro evidence suggesting that fruit and vegetable $\mathrm{AO}$ can provide protection against oxygen radicals. However much more information is needed regarding in vivo uptake and metabolism of these compounds, and wherever possible, evidence of $\mathrm{AO}$ protection using in vivo or ex vivo biomarkers of oxidative damage (de Zwart et al., 1999). This type of information will provide further evidence necessary to establish a role between $\mathrm{AO}$ phytochemicals and human health.

\section{Fruit and vegetable antioxidants}

Although plants synthesize AO that are used in their own defense against oxidative stress, certain types of $\mathrm{AO}$ are present in sufficient abundance to make a significant contribution to the $\mathrm{AO}$ complement found in a diet of fruit and vegetables. The major classes of $\mathrm{AO}$ phytochemicals include ascorbate, carotenoids, tocopherols, and phenolics; and for the latter three classes, numerous structural variations exist. Phenolics are probably the largest and most diverse group of plant food $\mathrm{AO}$, with several thousand unique types reported (Machiex et al., 1990). Phenolics are synthesized by the shikimic acid and phenylpropanoid pathways and include various major groups. These phenolic groups are distinguished by their number of carbon atoms, and by the structure of the parent molecule. In contrast to other AO found in fruit, such as carotenoids and tocopherols which are lipid soluble, phenolic AO are water soluble and are therefore likely to serve in the aqueous environment of the cell. Phenolics act as AO by scavenging free radicals, terminating peroxy-radical formation, chelating transition metal ions, and by working with ascorbate in oxidative defense.

\section{Fruit phenolics}

Two major classes of phenolics are common to small fruit species, namely the flavonoids and non-flavonoids. Of the non-flavonoid components, the hydroxycinnamate esters, especially chlorogenic acid, are predominant in many small fruit species. Other non-flavonoid phenolics include hydroxycinnamic acids and simple phenolics. The flavonoids include several major subclasses, of which anthocyanins are probably the best known since they are the pigments which impart red, purple, and blue coloration to fruit. Other small fruit flavonoids include flavonols (and their glycosides), catechins (flavan monomers), and proanthocyanidins (condensed tannins) (Fig. 1).

\section{Methods for the measurement of AO capacity, phenolics, and anthocyanins}

There are numerous methods for the measurement of antioxidant capacity. Data reported herein were obtained using the oxygen radical absorbing capacity (ORAC) assay (Cao et al., 1993, 1995) on crude fruit extracts. The ORAC assay has been applied to the estimation of total AO capacity in complex foods, blood serum, urine, and pure compounds (CaO and Prior, 1999). The ORAC assay is an aqueous assay and therefore well suited to water soluble components. ORAC values have been obtained for numerous fruit and vegetables (Cao et al., 1996; Kalt et al., 1999a; Prior et al., 1998; Wang et al., 1996; Wang and Lin, 2000). In the fruit species examined, ORAC was strongly and positively correlated with phenolic and anthocyanin content (Prior et al., 1998; Kalt et al., 1999a). The ORAC values of selected foods were similar to values reported using other methods for measuring AO capacity, including the chemiluminescent assay, and the trolox equivalents antioxidant capacity (TEAC) assay (Prior and Cao, 2000).

Estimates of total phenolic content are commonly obtained using the Folin-Ciocalteu method. This colorimetric method is based on development of a chromogen in proportion to the number of oxidizable groups in the sample components. Since phenolics represent a widely diverse group of compounds, which have various numbers of oxidizable groups, differences in extinction coefficient $(\varepsilon)$ values occur. Gallic acid $(\varepsilon=23.3)$ is often used as a standard, however in an evaluation

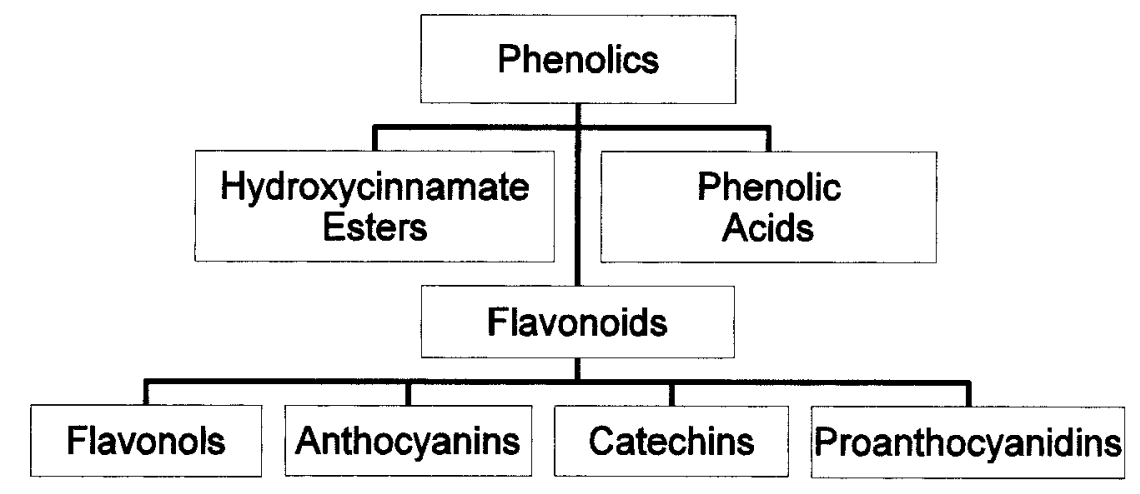

Fig. 1. Organization of phenolic components commonly found in small fruit, including blueberry. 
of the Folin-Ciocalteu method, $\varepsilon$ values for 14 pure phenolic compounds ranged between 0.8 and 57 (Singleton and Rossi, 1965). Ascorbate can contribute to color development by FolinCiocalteu reagents (Singleton and Rossi, 1965). In spite of these limitations, this method is a widely accepted estimator of total dissolved phenolics.

Total anthocyanins can be estimated colorimetrically using the $\mathrm{pH}$ differential method (Wrolstad, 1976). Using two buffers, the $\mathrm{pH}$-dependent ionic forms of anthocyanins can be controlled and used to assess their absorptivity. Results are expressed using an $\varepsilon$ value of a single anthocyanin, although many may be present in the sample. Wrolstad (1976) reports $\varepsilon$ values for pure anthocyanins in various solvents ranging from 795 to 41,700 , with most $\varepsilon$ values ranging between 20,000 to 30,000 . Using potassium metabisulfite, this method can also be used to account for the color created by phenolic condensation products. These condensation products typically arise after tissue disruption and oxidation.

\section{Horticultural factors affecting fruit phenolics and $A O$ capacity}

The purported health benefits of dietary $\mathrm{AO}$ are an incentive to study small fruit phenolic composition with an eye to identifying and retaining $\mathrm{AO}$ quality. Production and processing factors which influence phenolic content and AO capacity will be reviewed with a special emphasis on blueberries (Vaccinium sp. L.) since both highbush (V. corymbosum L.) and lowbush blueberries ( $V$. angustifolium Aiton) are notable for their high $\mathrm{AO}$ capacity (Wang et al., 1996; Prior et al., 1998; Kalt et al., 1999a).

\section{ORAC among genera}

Among three surveys conducted using the ORAC assay (Cao et al., 1996; Wang et al., 1996; Prior et al., 1998 ) blueberry had the highest ORAC among over 30 fruit and vegetables examined. In their survey of blueberry species, Prior et al. (1998) found ORAC strongly and positively correlated with both the total phenolic content $(r=0.85)$ and anthocyanin content $(r=0.77)$ of the fruit. A compilation of published results of seven small fruit species representing three genera illustrated a similar pattern (Fig. 2A and B). All of these fruit crops possess a red to blue color when mature, indicating that anthocyanins constitute a portion of the phenolic complement in these fruit.
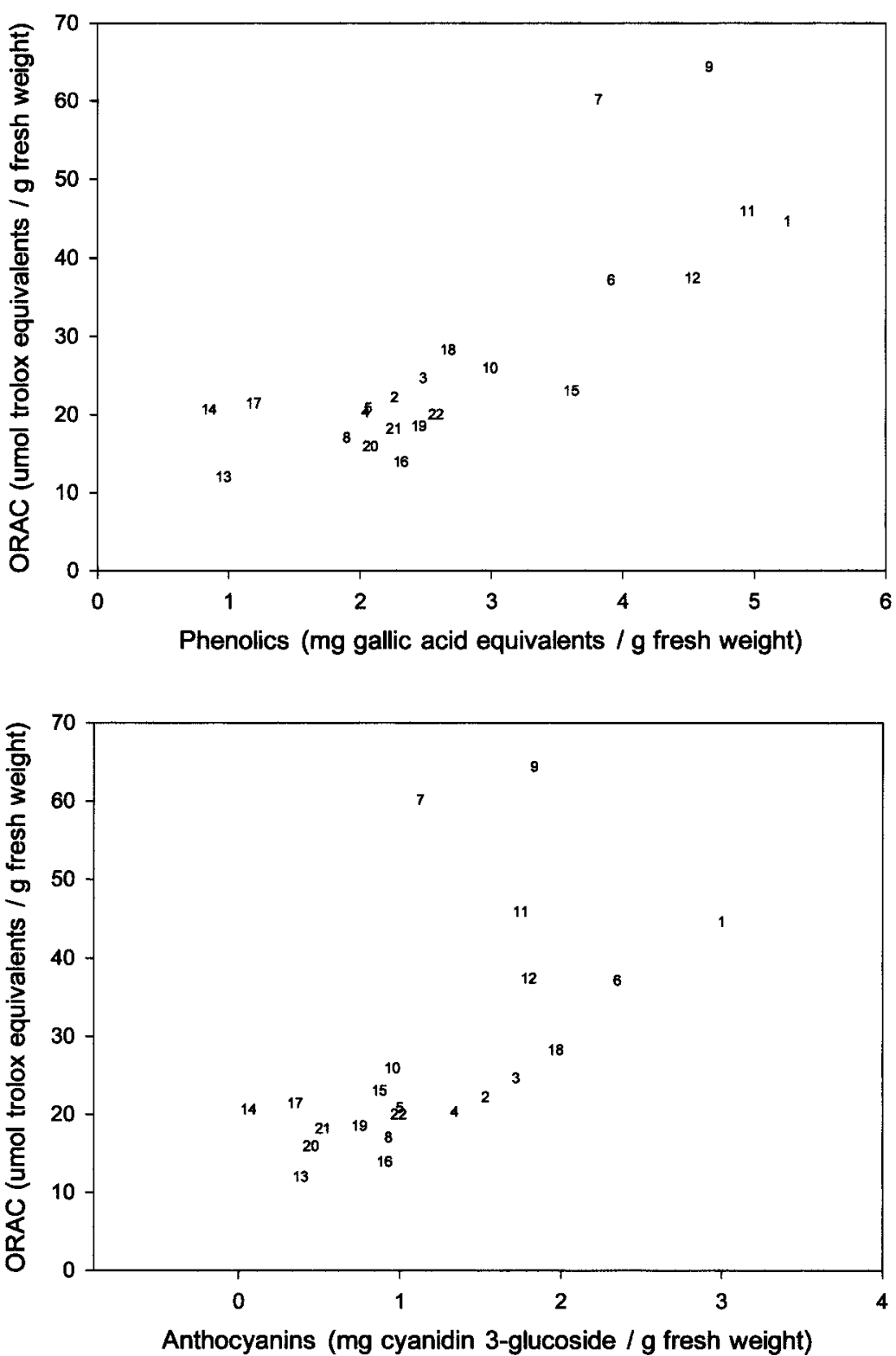

Fig. 2. (A) Relationship between total phenolic content and oxygen radical absorbance capacity (ORAC) of small fruit. 1) bilberry (Vaccinium myntillus L.) ${ }^{z}$; 2) blackberry (Rubus L.) 'Chester Thornless'; 3) blackberry 'Hull Thornless'; 4) blackberry 'Triple Crown', 5) highbush blueberry (V. conymbosum L.) 'Jersey"; 6) highbush blueberry

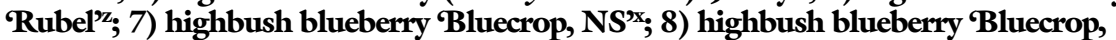
$M^{\prime 2}$; 9) lowbush blueberry (V. angustifolium Aiton), NS ; 10) lowbush blueberry, $\left.M^{z} ; 11\right)$ lowbush blueberry, NS z; 12) lowbush blueberry, PEI $^{z}$; 13) strawberry (Fragaria $\times$ ananassa Duch.) 'Allstar’y; 14) strawberry Kent $^{\text {’x}}$; 15) rabbiteye blueberry (V. asbeii Reade) 'Tifbluez; 16) rabbiteye blueberry 'Climax'z; 17) raspberry (Rubus idaeus L.) 'Nova'; 18 ) raspberry (Rubus occidentalis L.) Jewel'y; 19) raspberry (Rubus idaeus L.)'Autumn Bliss'; 20) raspberry 'Canby'; 21) raspberry 'Sentry'; 22) rasp-

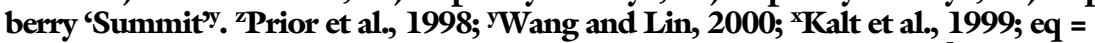
equivalents, FW = fresh weight, GAE = gallic acid equivalents; $1 \mathrm{mg} \cdot \mathrm{g}^{-1}=1000$ ppm. (B) Relationship between anthocyanin content and oxygen radical absorbing capacity (ORAC) of small fruit. Numbers denote the same species as in A; C-3G = cyanidin 3-

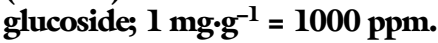


lowbush blueberries, and bilberries, ORAC extended over a 1.8-fold range. The extent to which phenolic content and ORAC can vary within the same cultivar is illustrated by the two samples of 'Bluecrop' (Fig. 2A and B, \#7 and 8). While anthocyanin content was quite similar between the two samples, total phenolic content and ORAC was substantially greater in the 'Bluecrop' from Nova Scotia (\#7). Lingonberry ( $V$. vitis ideae L.), which have a substantially lower pigment content than blueberry species, had a high ORAC due to its high level of total phenolics (Kalt, unpublished).

\section{ORAC among lowbush blueberry genotypes}

Commercial lowbush blueberries are made up of hundreds and possibly thousands of unique genotypes. Phenotypic differences in fruit size, plant height and leaf color make clones easy to distinguish in commercial lowbush blueberry fields. Differences among individual clones are particularly apparent during the autumn when leaf colors range from orange to maroon. In a survey of 135 clones of wild lowbush blueberries, both total phenolic and anthocyanin content varied over about a 3.5-fold range while ORAC spanned an about 5 -fold range (Fig. 3) (Duy, 1999). Phenotypic variability of this magnitude suggests that genotypes may differ substantially with respect to their phenolic biosynthetic potential and that it may be possible to optimize these traits using conventional or biotechnological breeding techniques. No correlation was found between the anthocyanin content of fruit and leaves in this survey.

\section{Anthocyanin profile and ORAC}

In ripe blueberry fruit, anthocyanins make a significant contribution to the ORAC as evidenced by the strong positive relationship between these two characteristics (Prior et al., 1998). However different anthocyanidin glycosides have different ORAC values (Wang et al., 1997). This raises a question whether the AO capacity of a standardized level of anthocyanins, could differ due to the specific anthocyanins present. Kalt et al. (1999b) described the anthocyanin profile of a selection of blueberry species and phenotypes and calculated their contribu- tion to ORAC. Among these blueberry species, the anthocyanin profile had relatively little impact on the anthocyanin contribution to the calculated ORAC. This may be expected since blueberry species have numerous types of anthocyanins, and thus no single anthocyanin would have a major influence on the ORAC. However in a fruit such as strawberry that contains almost exclusively pelargonidin and cyanidin glycosides, phenotypes containing a greater proportion of cyanidin 3-glucoside [ORAC $=2.24$ (Wang et al., 1997)] may have a higher total ORAC due to anthocyanins, than genotypes with a greater proportion of pelargonidin 3-glucoside [ORAC = 1.54 (Wang et al., 1997)]. In cultivated strawberries (Fragaria Xananassa Duch.) the pelargonidin 3-glucoside to cyanidin 3 -glucoside ratio is about 20:1, while in woodland strawberry (Fragaria vesca L.) this ratio is closer to 1:1 (Mazza and Miniati, 1993).

\section{Geographical variation}

ORAC was measured in mixtures of multitudinous clones of lowbush blueberries that had been harvested during three growing seasons, from regions where they are commercially produced (i.e., Canadian provinces east of Ontario and the state of Maine). These regions vary in their climatic conditions as illustrated by their differences in growing degree days (Gordon and Bootsma, 1993). In a separate analysis of each of the 3 years, there were significant differences in the ORAC values of fruit from different regions. Blueberries from Newfoundland, which has the coldest growing conditions, had the highest ORAC in 2 of the 3 years of study. Anthocyanin production can be stimulated by cold growing conditions (Nozzolillo et al., 1990; Saure, 1990). Biotic conditions (e.g., herbivory, disease) and abiotic conditions (temperature, moisture, irradiation, soil fertility) can vary markedly from year to year and affect the content of phenolic components (Jones and Hartley, 1999). Several more years of study are required to determine whether geographical differences in ORAC are consistent.

\section{Year-to-year differences in blueberry phenolics}

Data are lacking on year-to-year differences in ORAC however annual differences in blueberry anthocyanins have been monitored. Since anthocyanins are strongly correlated to the AO capacity in blueberries (Prior et al., 1998), year-to-year variation in anthocyanin content could be an indicator of similar variation in ORAC. Sea-
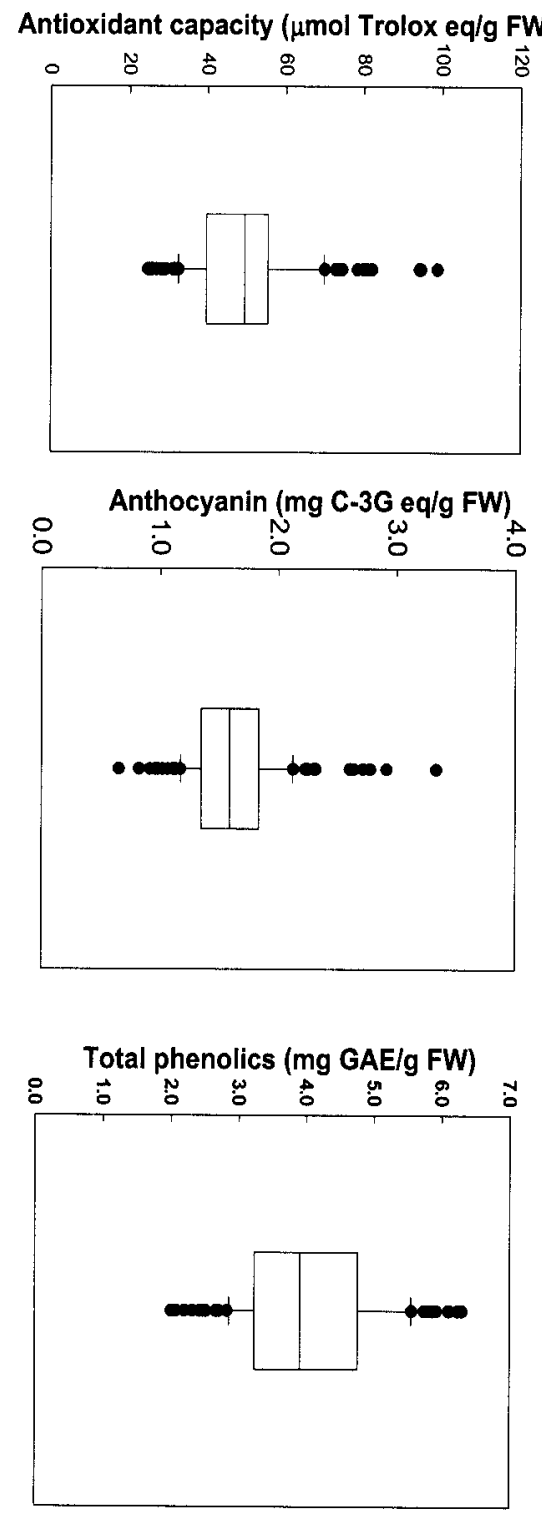

Fig. 3. Variation in oxygen radical absorbing capacity, anthocyanin content and total phenolic content of 135 clones of lowbush blueberries. The median is indicated by the mid line of the shaded box, while the $75^{\text {th }}$ and $25^{\text {th }}$ percentiles are the upper and lower boundaries, respectively, of the box. Error bars indicate the $\mathbf{9 0}^{\text {th }}$ and $10^{\text {th }}$ percentiles, and all points which lie outside these percentiles are indicated by dots; eq = equivalents, FW = fresh weight, $\mathbf{C - 3 G}=$ cyanidin 3-glucoside, $\mathbf{G A E}=$ gallic acid equivalents, $1.0 \mathrm{mg} \cdot \mathrm{g}^{-1}=1000 \mathrm{ppm}$. 
sonal variation in the anthocyanin content among lowbush blueberry cultivars was quite marked in fruit harvested from the same site, over seven seasons, when the results of various studies were compared (Kalt and McDonald, 1996; Kalt et al., 1999; Kalt, unpublished). Anthocyanin content varied by up to 2.4 -fold for 'Blomidon', 1.8-fold for 'Cumberland', and 2.0-fold for 'Fundy' fruit during this period. Although year-toyear variation was substantial, the relative ranking was fairly consistent, with 'Fundy' greater than the other two cultivars in all years examined. Again, a multitude of environmental factors are known to influence both the phenolic content of leaves and fruit (Jones and Hartley, 1999), thus many years of measurement of anthocyanin and other phenolics, and ORAC are needed to assess the potential magnitude of seasonal variation in these parameters.

\section{Fruit maturity}

Prior et al. (1998) reported higher ORAC of rabbiteye blueberry fruit of more advanced ripeness. Wang and Lin (2000) reported the highest ORAC in red-ripe raspberries (Rubus idaeus L.), as compared to two less ripe stages. However, in blackberries (Rubus L.) and strawberries values were lower at more advanced stages of ripeness. Kalt et al. (2000a) found no differences in ORAC among five ripeness stages of three highbush blueberry cultivars. The ripeness stages ranged from $0 \%$ to $50 \%$ pink to fully ripe, with anthocyanin content ranging from almost 0 in the least ripe, to about $9 \mathrm{mg}$ cyanidin 3glucoside equivalents per gram fresh weight $(9000 \mathrm{ppm})$ in the most ripe fruit. The total phenolic content was slightly lower in fruit of more advanced ripeness, possibly due to a decline in proanthocyanidins. A decline in proanthocyanidins with advanced maturity has been reported for apples (Malus domestica Borkh.) (Lister et al., 1994). These results suggest that, although they are different in their phenolic profile, underripe highbush blueberries may be as good as fully ripe fruit with respect to ORAC.

\section{Effects of storage and processing}

Strawberries, raspberries, and highbush and lowbush blueberries were stored at temperatures between 0 to $30{ }^{\circ} \mathrm{C}\left(32.0\right.$ to $\left.86.0{ }^{\circ} \mathrm{F}\right)$ for up to $8 \mathrm{~d}$ to determine the effect of storage time and temperature on fruit $\mathrm{AO}$ capacity. The ORAC and anthocyanin content of strawberries and raspberries increased during storage at temperatures $>0{ }^{\circ} \mathrm{C}\left(32^{\circ} \mathrm{F}\right)$. There was also an increase in the total phenolic content of raspberries. There was no change in the ORAC of either blueberry species, although there was a slight increase in the pigment content of the highbush fruit when stored at $20^{\circ} \mathrm{C}\left(86^{\circ} \mathrm{F}\right)$ (Kalt et al., 1999a).

Many fruit-based foods are processed into products such as beverages, baked goods or confectionaries. The processing treatments that fruit undergo may have a detrimental effect on their phytochemical AO. Processing parameters, including heat, aeration, and neutral $\mathrm{pH}$ were found to significantly decrease the $\mathrm{AO}$ capacity of blueberries. While a higher temperature $\left[60^{\circ} \mathrm{C}\left(140{ }^{\circ} \mathrm{F}\right)\right]$ initially appeared to extract more phenolics and ORAC, samples showed damage after a storage period. During storage, anthocyanins, total phenolics and ORAC declined in the samples extracted at $60{ }^{\circ} \mathrm{C}$, but not in samples extracted at $25^{\circ} \mathrm{C}\left(77.0^{\circ} \mathrm{F}\right)$. Numerous processed blueberry products were surveyed and in general, ORAC was lower in products that had undergone more extensive processing. Among the processed products surveyed, there was a strong positive correlation between monomeric anthocyanins and ORAC $\left(R^{2}=0.92\right)$ and total phenolics and ORAC $\left(R^{2}=0.95\right)$. However, the percent polymeric color, which is an indicator of phenolic damage, was negatively correlated with ORAC $\left(R^{2}\right.$ $=-0.64)$ (Kalt et al., 2000b).

\section{Future research}

Evidence to date indicates that phenolic components, including anthocyanins, make a substantial contribution to the dietary $\mathrm{AO}$ contained in many small fruit crops. These compounds serve a variety of ecological functions in plants, and their content in fruit can vary substantially in response to numerous biotic and abiotic factors, many of which are as yet poorly understood. The production of phenolic components can be highly variable, therefore opportunities exist to enhance their content through genetic selection, and pre- and postharvest environmental manipulation. Future research on other phenolic compo- nents is also warranted, since these compounds [e.g., catechins, proanthocyanidins (Fig. 1)] are not well characterized in small fruit species, and may be at least as important as anthocyanins with respect to their human health functionality.

\section{Literature cited}

Ames, B.M.., M..K. Shigena, and T.M. Hagen. 1993. Oxidants, antioxidants and the degenerative disease of aging. Proc. Natl. Acad. Sci. USA 90:7915-7922.

Block, G., B. Patterson, and A. Subar. 1992. Fruits, vegetables and cancer prevention: A review of epidemiological evidence. Nutr. Cancer 18:1-29.

Cantuti-Castelvetri I., B. Shukitt-Hale, and J.A. Joseph. 2000. Neurobehavioural aspects of antioxidants in aging. Intl. J. Dev. Neurosci. 18:367-381.

Cao, G., H.M. Alessio, and R.G. Cutler. 1993. Oxygen-radical absorbance capacity assay for antioxidants. Free Radical Biol. Med. 14:303-311.

Cao, G., E. Sofic, and R.L. Prior. 1996. Antioxidant capacity of tea and common vegetables. J. Agr. Food Chem. 44: 34263431 .

Cao, G., C.P. Verdon, A.H.B. Wu, H. Wang, and R.L. Prior. 1995. Automated oxygen radical absorbance capacity assay using the COBAS FARA II. Clin. Chem. 41:1738-1744.

Cao, G. and R.L. Prior. 1999. Measurement of oxygen radical absorbance capacity in biological samples. In: L. Packer (ed.). Meth. Enzymol. 299. Oxidants and antioxidants Part A. Academic Press, London.

de Zwart, L.L., J.H.N. Meerman, J.N.M. Commandeur, and N.P.E. Vermeulen. 1999. Biomarkers of free radical damage: applications in experimental animals and humans. Free Radical Biol. Med. 26:202226.

Duy, J.C. 1999. A survey of the quantitative intraspecific variation of anthocyanins, phenolics and antioxidant capacity in leaves and fruit of Vaccinium angustifolium Aiton clones in Nova Scotia. MSc thesis. Acadia Univ., Wolfville, Nova Scotia.

Fuller, C.J. and I. Jialal. 1997. Antioxidants and LDL oxidation, p. 115-130. In: H.S. Garewal (ed.). Antioxidants and disease prevention. CRC Press, Boca Raton, Fla.

Gordon, R. and A. Bootsma. 1993. Analyses of growing degree-days for agriculture in Atlantic Canada. Clim. Res. 3:169-176.

Hertog, M.G.L., E.J.M. Feskens, P.C.H. 
Hollman, M.B. Katan, and D. Kromhout. 1993. Dietary antioxidant flavonoids and risk of coronary heart disease: The Zutphen Elderly Study. The Lancet 342:1007-1011.

Jones, C.G. and S.E. Hartley. 1999. A protein competition model of phenolic allocation. Oikos 86:27-44.

Joshipura, K.J., A. Ascherio, J.E. Manson, M.J. Stampfer, E.B. Rimm, F.E. Speizer, C.H.Hennekens, D. Spiegelman, and W.C. Willett. 1999. Fruit and vegetable intake in relation to risk of ischemic stroke. J. Amer. Med. Assn. 282(13):1233-1239.

Kalt, W. and J.E. McDonald. 1996. Chemical composition of lowbush blueberry cultivars. J. Amer. Soc. Hort. Sci. 121:142146.

Kalt, W., C.F. Forney, A. Martin, and R.L. Prior. 1999a. Antioxidant capacity, vitamin C, phenolics, and anthocyanins after fresh storage of small fruits. J. Agr. Food Chem. 47:4638-4644.

Kalt, W., J.E. McDonald, R.D. Ricker, and X. Lu. 1999b. Anthocyanin content and profile within and among blueberry species. Can. J. Plant Sci. 79:617-623.

Kalt, W., C. Lawand, and C.F. Forney. 2000a. Antioxidant capacity and anthocyanin and phenolic content of highbush blueberries of different maturities. HortScience 35:394.

Kalt, W., J.E. McDonald, and H. Donner. $2000 \mathrm{~b}$. Anthocyanins, phenolics and antioxidant capacity of processed blueberry products. J. Food Sci. 65:390-393.

Key, T.J.A., M. Thorogood, P.N.Appleby, and M.L. Burr. 1996. Dietary habits and mortality in 11000 vegetarians and health conscious people: results of a 17 year follow up. Brit. Med. J. 313:775-779.

Lister, C.E., J.E. Lancaster, and K.H. Sutton. 1994. Developmental changes in the concentration and composition of flavonoids in skin of a red and a green apple cultivar. J. Sci. Food Agr. 64:155-161.

Machiex, J.-J., A. Fleuriet, and J. Billot. 1990. Phenolic composition of individual fruits, p. 105-148. In: Fruit phenolics. CRC Press, Boca Raton, Fla.
Mazza G. and E. Miniati. 1993. Small fruits, p. 108. In: Anthocyanins in fruits, vegetables and grains. CRC Press, Boca Raton, Fla.

Ness, A.R. and J.W. Powles. 1997. Fruit and vegetables, and cardiovascular disease: A review. Inter. J. Epidemiol. 26(1):1-13.

Nozzolillo, C.P. Isabelle and G. Das. 1990. Seasonal changes in the phenolic constituents of jack pine seedlines (Pinusbanksiana) in relation to the purpling phenomenon. Can. J. Bot. 68:2010-2017.

Prior, R.L., G. Cao, A. Martin, E. Sofic, J. McEwen, C. O'Brien, N. Lischner, M. Ehlenfeldt, W. Kalt, G. Krewer, and C.M. Mainland. 1998. Antioxidant capacity as influenced by total phenolic and anthocyanin content, maturity and variety of $\mathrm{Vac}$ cinium species. J. Agr. Food Chem. 46:2686-2693.

Prior, R.L. and G. Cao. 2000. Antioxidant phytochemicals in fruits and vegetables: Diet and health implications. HortScience 35:588-592.

Saure, M.C. 1990. External control of anthocyanin formation in apple. Scientia Hort. 42:181-218.

Singleton, V.L. and J.A. Rossi. 1965. Colorimetry of total phenolics with phosphomolybdic-phosphotungstic acid reagents. Amer. J. Enol. Viticult. 16:144-158.

Steinmetz K.A. and J.D. Potter. 1996. Vegetables, fruit, and cancer prevention: A review. J. Amer. Diet. Assn. 96( 10 ):10271039.

Wang, H., G. Cao, and R.L. Prior. 1996. Total antioxidant capacity of fruits. J. Agr. Food Chem. 44:701-705.

Wang, H., G. Cao, and R.L. Prior. 1997. Oxygen radical absorbing capacity of anthocyanins. J. Agr. Food Chem. 45:304309.

Wang, S.Y. and H.-S. Lin. 2000. Antioxidant activity in fruits and leaves of blackberry, raspberry, and strawberry varies with cultivar and developmental stage. J. Agr. Food Chem. 48:140-146.

Wrolstad, R.E. 1976. Color and pigment analysis in fruit products. Oreg. State Univ. Agr. Expt. Sta., Corvallis, Bul. 624. 